\title{
THE NUTRITIVE VALUES OF SOME FORAGE CROPS OF PUERTO RICO
}

\section{Grasses.}

\section{Corrections:}

On page 106, the third line of the heading of Table 2 should read: "and Body Nitrogen in Urine per 1000 Grams of Body Weight, Based".

On page 107, under the column headed by "Body Nitrogen in Urine Per Kilogram of Body Weight"' should read 0.535 instead of 0.801 and 0.372 instead of 0.335 . Under the column headed by "Average Body Nitrogen in Urine Per Kilogram of Body Weight"' should read 0.561 instead of 0.526 .

TABLE A

SCHEDULE'FOLLOWED STARTING SEPTEMBER 22, 1937 TO MARCH 29, 1938

\begin{tabular}{|c|c|c|c|c|c|c|}
\hline \multirow{2}{*}{$\begin{array}{l}\text { Trial } \\
\text { No. }\end{array}$} & \multirow{2}{*}{ Ration } & \multicolumn{2}{|c|}{$\begin{array}{l}\text { Preliminary } \\
\text { Period }\end{array}$} & \multicolumn{2}{|c|}{$\begin{array}{l}\text { Experimental } \\
\text { Period }\end{array}$} & \multirow{2}{*}{$\begin{array}{l}\text { Animal } \\
\text { Number }\end{array}$} \\
\hline & & Starts & Ends & Starts & Ends & \\
\hline $3 .$. & $\begin{array}{l}\text { Soy Bean (in flower) } \mathrm{N}-\mathrm{P}-\mathrm{K} \\
\text { Otootan variety........... }\end{array}$ & Oet. 12. & Oct. 22 . . & Oct. $22 \ldots$ & Nov. 1 . . & $16-17-18-20-21$ \\
\hline $4 \ldots \ldots \ldots$ & $\begin{array}{l}\text { Soy Bean (in flower) } \mathrm{N}-\mathrm{P}-\mathrm{K} \\
\text { Otootan variety........... }\end{array}$ & Oct. 22. & Nor. $1 .$. & Nov. 1.. & Nov. 11. & $40-41-42-43-44$ \\
\hline $5 \ldots \ldots \ldots$ & $\begin{array}{l}\text { Soy Bean (in full-pods) } \mathrm{N}- \\
\mathrm{P}-\mathrm{K} \\
\text { Otootan variety ............. }\end{array}$ & Nov. 1.. & Nov. 11 , & Nov. 11. & Nov. 21. & $16-17-18-20-21$ \\
\hline $6 \ldots \ldots$ & $\begin{array}{l}\text { Soy Bean (in full-pods) } \mathrm{N}- \\
\text { P-K } \\
\text { Otootan variety............. }\end{array}$ & Nov. 11. & Nov. 21. & Nov. 21. & Dec. 1.. & $40-41-42-43-44$ \\
\hline $7 \ldots \ldots \ldots$ & $\begin{array}{l}\text { Velvet Bean (in flower) } \mathrm{N}- \\
\mathrm{P}-\mathrm{K} \ldots \ldots \ldots \ldots \ldots \ldots\end{array}$ & Nov. 21. & Dec. $1 \ldots$ & Dec. $1 \ldots$ & Dec. 11.. & $16-18-20-21$ \\
\hline $8 \ldots \ldots \ldots$ & $\begin{array}{l}\text { Velvet Bean (in flower) } \mathrm{N}- \\
\mathrm{P}-\mathrm{K} . \ldots \ldots \ldots \ldots \ldots \ldots\end{array}$ & Dec. 1.. & Dec. 11 & Dec. 11. & Dec. 21. & $40-41-42-44$ \\
\hline $9 \ldots \ldots$ & $\begin{array}{l}\text { Velvet Bean (in full-pods) } \\
\mathrm{N}-\mathrm{P}-\mathrm{K} \ldots \ldots \ldots \ldots \ldots \ldots\end{array}$ & Jan. $2 \ldots$ & Jan. $12 .$. & Jan. $12 \ldots$ & Jan. 22. & $16-17-18-19-20-21$ \\
\hline $10 \ldots \ldots \ldots$ & $\begin{array}{c}\text { Pigeon Pea (before blooming) } \\
\text { N-P-K } \ldots \ldots \ldots \ldots \ldots \ldots \ldots \ldots\end{array}$ & Jan. 22.. & Feb. $1 \ldots$ & Feb. $1 \ldots$ & Feb. 11. & $16-17-18-19-20-21$ * \\
\hline $11 \ldots \ldots \ldots$ & $\begin{array}{l}\text { Pigeon Pea (before blooming) } \\
\text { N-P-K } \ldots \ldots \ldots \ldots \ldots \ldots\end{array}$ & Feb. 1.. & Feb. 11. & Eeb. 11 & Feb. 21 & $16-17-18-19-20-21$ \\
\hline $\begin{array}{l}1 \\
2 \ldots \ldots \ldots\end{array}$ & $\begin{array}{l}\text { Yaragua, } \mathrm{N} \text { and } \mathrm{P} \ldots \ldots \ldots \\
\text { Yaragua, } \mathrm{N} \text { and } \mathrm{P} \ldots \ldots \ldots\end{array}$ & $\begin{array}{l}\text { Sept. } 22 . \\
\text { Ozt. } 2 \ldots\end{array}$ & $\begin{array}{l}\text { Oct. } 2 \ldots \\
\text { Oct. } 12 \ldots\end{array}$ & $\begin{array}{l}\text { Oct. } 2 \ldots \\
\text { Oct. } 12 \ldots\end{array}$ & $\begin{array}{l}\text { Oct. } 12 \ldots \\
\text { Oct. } 22 \ldots\end{array}$ & $\begin{array}{l}16-17-18-20-21^{* *} \\
40-41-42-43-44\end{array}$ \\
\hline $12 \ldots \ldots \ldots$ & Merker Grass ( $\mathrm{N}$ only).... & Feb. 11. & Feb. 21. & Feb. 2!. & Mar. 3.. & $1-3-4-40-41$ \\
\hline $13 .$. & Merker Grass ( $N$ only).... & Feb. 21 . & Mar. 3.. & Mar. 4.. & Mar. 14. & $16-17-18-19-20-21$ \\
\hline $14 \ldots \ldots \ldots$ & $\begin{array}{c}\text { Merker Grass (N only) } 11 / 4+ \\
\text { Pigeon Pea (before } \\
\text { blooming) } 1 \ldots \ldots \ldots \ldots \ldots \ldots\end{array}$ & Mar. 9.. & Mar. 18. & Mar. 19. & Mar. 29. & $1-3-4-5-6-41$ \\
\hline
\end{tabular}

*All animals in preliminary period.

**This trial is not included in the calculations. 
464 THE JOURNAL OF AGRICULTURE OF THE UNIVERSITY OF P. R.

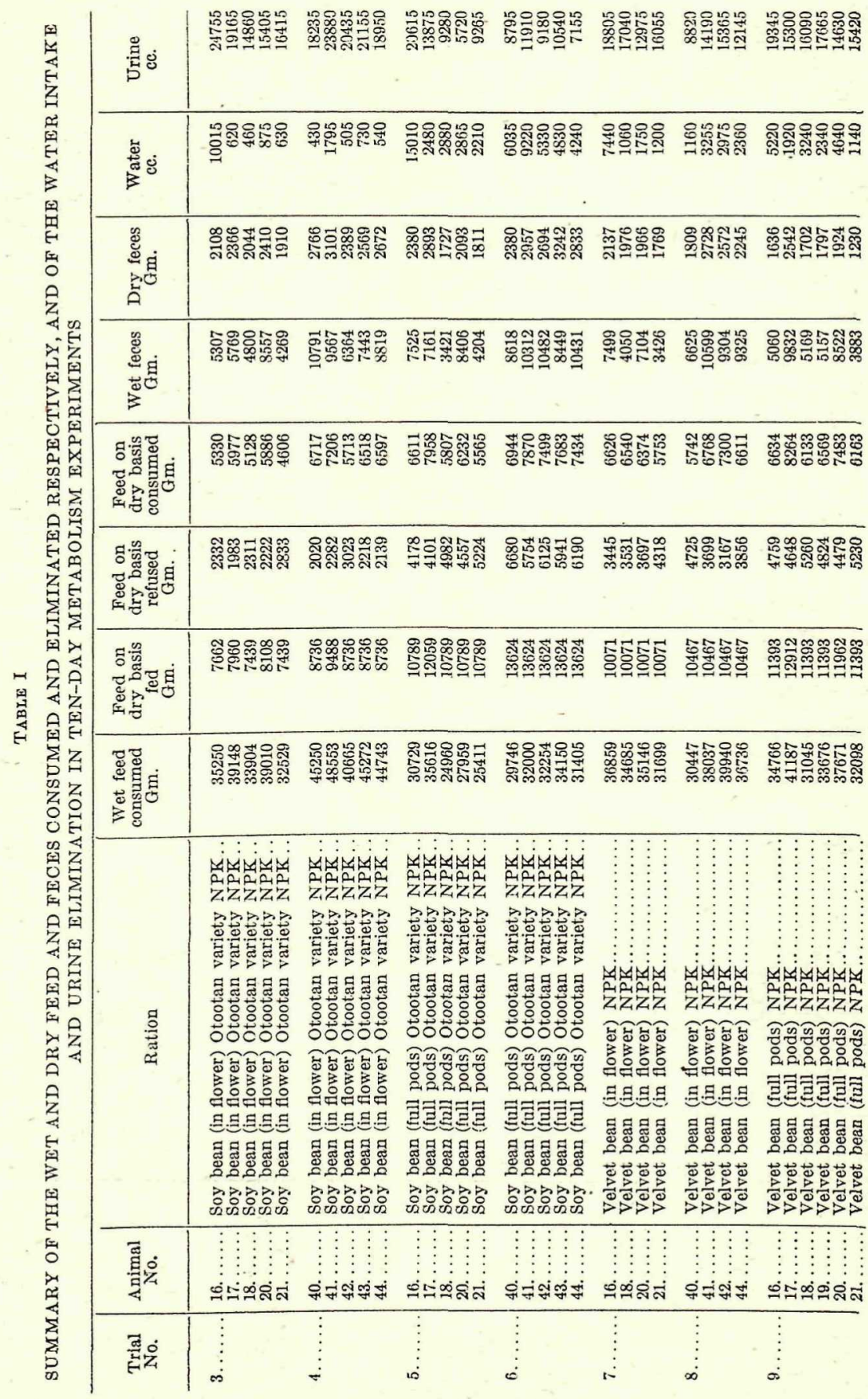




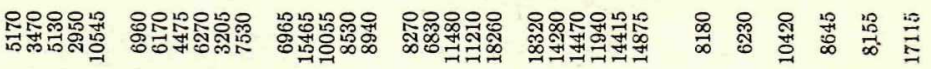

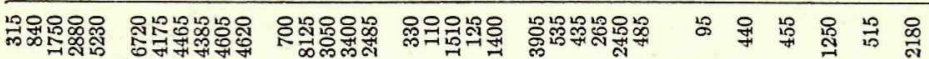

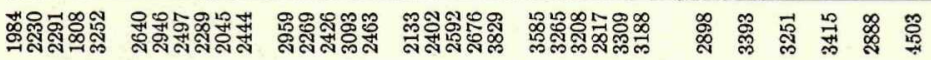

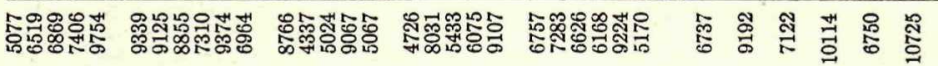

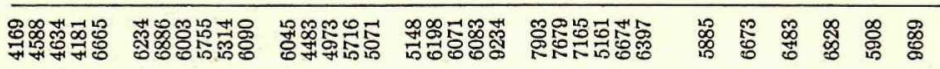

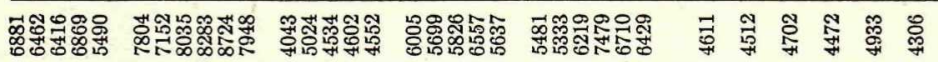

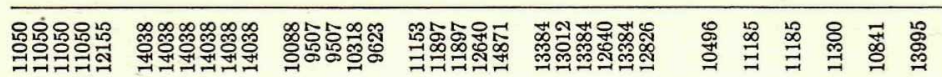

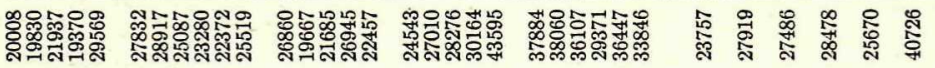

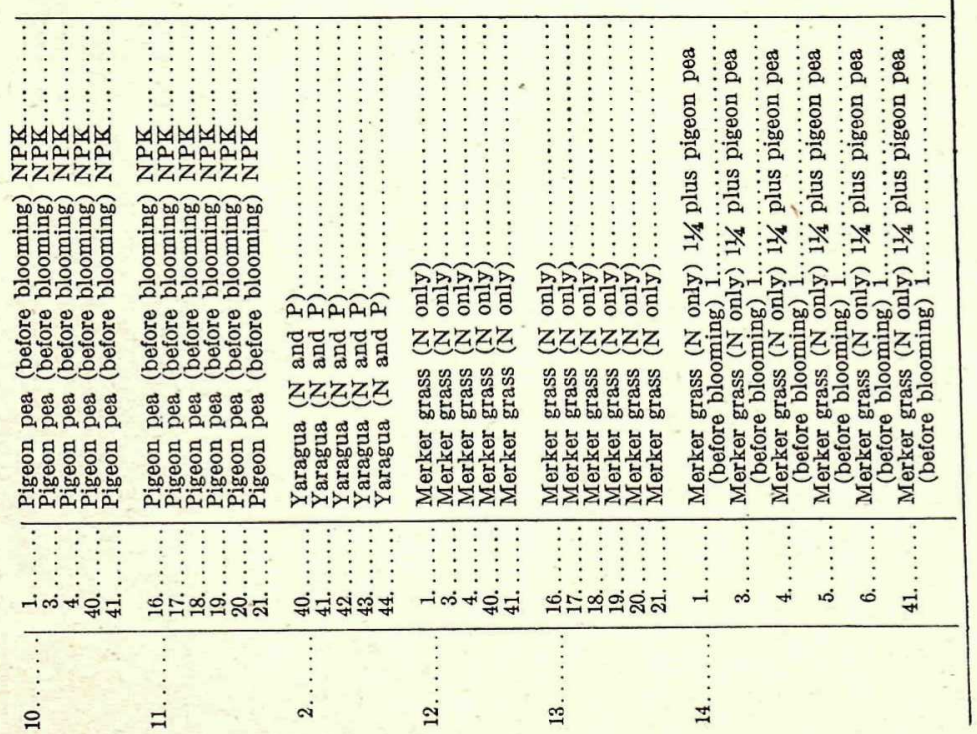


TABLE II

PER CENT NITROGEN IN FEED FED, REFUSE LEFT, FECES AND URINE (FEED, REFUSE AND FECES ANALYSES, PER CENT ON DRY BASIS) (URINE ANALYSES, PER CENT BY VOLUME)

\begin{tabular}{|c|c|c|c|c|c|c|}
\hline $\begin{array}{l}\text { Trial } \\
\text { No. }\end{array}$ & Ration & $\begin{array}{l}\text { Nitrogen } \\
\text { in } \\
\text { feed fed } \\
q_{q}\end{array}$ & $\begin{array}{l}\text { Nitrogen } \\
\text { in refuse } \\
\text { left } \\
\frac{6}{6}\end{array}$ & $\underset{\text { No. }}{\text { Animal }}$ & $\begin{array}{l}\text { Nitrogen } \\
\text { in feces } \\
q\end{array}$ & $\begin{array}{l}\text { Nitrogen } \\
\text { in } \underset{\%}{\%}\end{array}$ \\
\hline $3 \ldots \ldots$ & $\underset{\text { variety }}{\text { Soy bean (in flower) Otootan.. }}$ & 2. 42 & 1. 64 & $\begin{array}{l}16 \\
17 \\
18 \\
20 \\
21\end{array}$ & $\begin{array}{l}1.71 \\
1.84 \\
1.93 \\
1.90 \\
2.00\end{array}$ & $\begin{array}{l}0.37 \\
0.61 \\
0.60 \\
0.65 \\
0.54\end{array}$ \\
\hline $4 \ldots \ldots$ & $\begin{array}{l}\text { Soy bean (in flower) Otootan. } \\
\text { variety }\end{array}$ & 2.66 & 1. 62 & $\begin{array}{l}40 \\
41 \\
42 \\
43 \\
44\end{array}$ & $\begin{array}{l}1.70 \\
1.67 \\
1.86 \\
1.70 \\
1.71\end{array}$ & $\begin{array}{l}0.56 \\
0.54 \\
0.57 \\
0.53 \\
0.53\end{array}$ \\
\hline $5 \ldots \ldots$ & $\begin{array}{l}\text { Soy bean (in full pods)....... } \\
\text { Otootan variety }\end{array}$ & 2. 60 & 1.55 & $\begin{array}{l}16 \\
17 \\
18 \\
20 \\
21\end{array}$ & $\begin{array}{l}1.83 \\
1.97 \\
2.13 \\
1.98 \\
2.40\end{array}$ & $\begin{array}{l}0.42 \\
0.82 \\
0.83 \\
1.07 \\
0.92\end{array}$ \\
\hline $6 \ldots \ldots$ & $\underset{\text { Soy bean (in full pods) ....... }}{\text { Otootan variety }}$ & 2. 58 & 1. 61 & $\begin{array}{l}40 \\
41 \\
42 \\
43 \\
44\end{array}$ & $\begin{array}{l}1.91 \\
2.11 \\
2.16 \\
1.84 \\
1.98\end{array}$ & $\begin{array}{l}0.79 \\
0.95 \\
1.05 \\
1.00 \\
1.29\end{array}$ \\
\hline $7 \ldots \ldots$ & Velvet bean (in flower)...... & 2. 48 & 1. 67 & $\begin{array}{l}16 \\
18 \\
20 \\
21\end{array}$ & $\begin{array}{l}2.23 \\
2.45 \\
2.40 \\
2.64\end{array}$ & $\begin{array}{l}0.45 \\
0.52 \\
0.65 \\
0.52\end{array}$ \\
\hline $8 \ldots \ldots$ & Velvet bean (in flower)...... & 2. 53 & 1.67 & $\begin{array}{l}40 \\
41 \\
42 \\
44\end{array}$ & $\begin{array}{l}2.69 \\
2.99 \\
2.61 \\
2.63\end{array}$ & $\begin{array}{l}0.74 \\
0.68 \\
0.65 \\
0.78\end{array}$ \\
\hline $9 \ldots \ldots$ & Velvet bean (full pods) NPK. & 2. 61 & 2. 05 & $\begin{array}{l}16 \\
17 \\
18 \\
19 \\
20 \\
21\end{array}$ & $\begin{array}{l}3.36 \\
3.58 \\
3.36 \\
3.06 \\
3.57 \\
3.69\end{array}$ & $\begin{array}{l}0.41 \\
0.45 \\
0.45 \\
0.41 \\
0.41 \\
0.53\end{array}$ \\
\hline $10 \ldots \ldots$ & $\begin{array}{l}\text { Pigeon peas (before blooming). } \\
\mathrm{N}-\mathrm{P}-\mathrm{K}\end{array}$ & 3. 42 & 3. 57 & $\begin{array}{r}1 \\
3 \\
4 \\
40 \\
41\end{array}$ & $\begin{array}{l}2.87 \\
2.69 \\
2.83 \\
2.55 \\
2.56\end{array}$ & $\begin{array}{l}1.42 \\
2.12 \\
1.58 \\
1.35 \\
1.17\end{array}$ \\
\hline $11 \ldots \ldots$ & $\begin{array}{l}\text { Pigeon peas (before blooming). } \\
\mathrm{N}-\mathrm{P}-\mathrm{K}\end{array}$ & 3.05 & 3.05 & $\begin{array}{l}16 \\
17 \\
18 \\
19 \\
20 \\
21\end{array}$ & $\begin{array}{l}2.61 \\
2.74 \\
2.83 \\
2.72 \\
2.44 \\
2.81\end{array}$ & $\begin{array}{l}1.07 \\
1.67 \\
1.86 \\
1.24 \\
1.92 \\
1.35\end{array}$ \\
\hline $2 \ldots \ldots$ & Yaragua $-\mathrm{N}$ and $\mathrm{P} . \ldots \ldots \ldots$ & 0.80 & 0.44 & $\begin{array}{l}40 \\
41 \\
42 \\
43 \\
44\end{array}$ & $\begin{array}{l}\text { 1. } 22 \\
\text { 1. } 34 \\
\text { 1. } 37 \\
\text { 1. } 21 \\
\text { 1. } 31\end{array}$ & $\begin{array}{l}0.19 \\
0.12 \\
0.24 \\
0.20 \\
0.19\end{array}$ \\
\hline $12 \ldots \ldots$ & Merker grass (N only)...... & 1.80 & 1. 33 & $\begin{array}{r}1 \\
3 \\
4 \\
40 \\
41\end{array}$ & $\begin{array}{l}1.92 \\
2.16 \\
1.85 \\
1.80 \\
1.67\end{array}$ & $\begin{array}{l}0.55 \\
0.66 \\
0.45 \\
0.49 \\
0.46\end{array}$ \\
\hline $13 \ldots \ldots$ & Merker grass ( $\mathrm{N}$ only)....... & 1. 56 & 1.05 & $\begin{array}{l}16 \\
17 \\
18 \\
19 \\
20 \\
21\end{array}$ & $\begin{array}{l}1.38 \\
1.47 \\
1.49 \\
1.40 \\
1.38 \\
1.41\end{array}$ & $\begin{array}{l}0.34 \\
0.39 \\
0.40 \\
0.42 \\
0.35 \\
0.45\end{array}$ \\
\hline $14 \ldots \ldots$ & $\begin{array}{l}\text { Merker grass ( } N \text { only) } 11 / 4 \ldots . \\
\text { Pigeon peas (before } \\
\text { blooming) } 1\end{array}$ & 1. 97 & 1.16 & $\begin{array}{r}1 \\
3 \\
4 \\
5 \\
6 \\
41 \\
\end{array}$ & $\begin{array}{l}2.50 \\
2.40 \\
2.51 \\
2.42 \\
2.57 \\
2.23 \\
\end{array}$ & $\begin{array}{l}0.56 \\
0.71 \\
0.48 \\
0.71 \\
0.58 \\
0.45 \\
\end{array}$ \\
\hline
\end{tabular}


TABLE III

MAINTENANCE REQUIREMENT OF LAMBS AVERAGING 16.96 KILOGRAMS IN WEIGHT, BODY NITROGEN IN FECES PER GRAM OF DRY MATTER INGESTED, AND BODY NITROGEN IN URINE PER KILOGRAM OF BODY WEIGHT, BASED ON TEN-DAY TRIALS DURING WHICH NEARLY NITROGEN-FREE RATIONS WERE FED

\begin{tabular}{|c|c|c|c|c|c|}
\hline Remarks & $\begin{array}{l}\text { Lamb } \\
\text { No. }\end{array}$ & $\begin{array}{l}\text { Body } \\
\text { Nitrogen in } \\
\text { Feces } \\
\text { Per Gram of } \\
\text { Dry Matter } \\
\text { Ingested }\end{array}$ & $\begin{array}{l}\text { Average Body } \\
\text { Nitrogen in } \\
\text { Feces } \\
\text { Per Gram of } \\
\text { Dry Matter } \\
\text { Ingested }\end{array}$ & $\begin{array}{c}\text { Body } \\
\text { Nitrogen in } \\
\text { Urine } \\
\text { Per Kilogram } \\
\text { of Body } \\
\text { Weight }\end{array}$ & $\begin{array}{l}\text { Average Body } \\
\text { Nitrogen in } \\
\text { Urine } \\
\text { Per Kilogram } \\
\text { of Body } \\
\text { Weight }\end{array}$ \\
\hline $\begin{array}{l}\text { Value obtained in the year } \\
1936 \text { to } 1937\end{array}$ & $\begin{array}{l}1 \mathrm{~A} \\
2 \mathrm{~A} \\
3 \mathrm{~A} \\
1 \mathrm{~B} \\
2 \mathrm{~B} \\
3 \mathrm{~B}\end{array}$ & $\begin{array}{l}0.0041 \\
0.0051 \\
0.0036 \\
0.0038 \\
0.0046 \\
0.0046\end{array}$ & 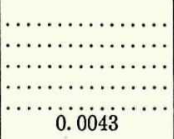 & $\begin{array}{l}0.535 \\
0.372 \\
0.441 \\
0.830 \\
0.626 \\
0.565\end{array}$ & 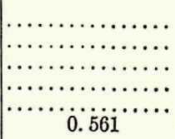 \\
\hline
\end{tabular}


THE JOURNAL OF AGRICULTURE OF THE UNIVERSTTY OF $P$. $R$.

\begin{tabular}{|c|c|c|c|c|c|c|c|c|}
\hline \multicolumn{3}{|c|}{ 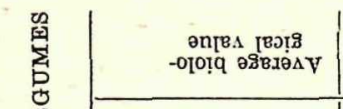 } & : & \% & : : & $\vdots \vdots$ & : & : \\
\hline 国 & & 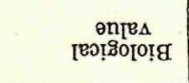 & ద్లేีడేణొలా & 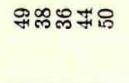 & 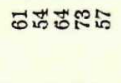 & 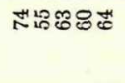 & 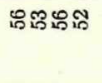 & 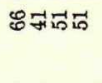 \\
\hline 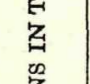 & & 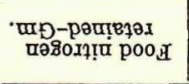 & 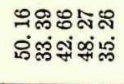 & 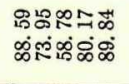 & 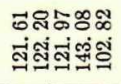 & 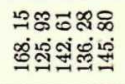 & 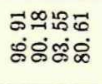 & 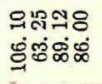 \\
\hline $\begin{array}{l}\text { 舅 } \\
0\end{array}$ & & 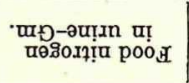 & 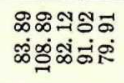 & 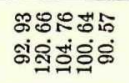 & 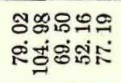 & 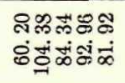 & 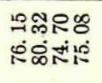 & 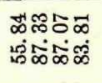 \\
\hline $\begin{array}{l}\text { Er } \\
\text { 帒 } \\
\text { on }\end{array}$ & & 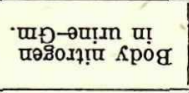 & 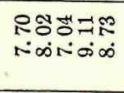 & 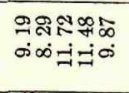 & 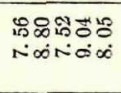 & 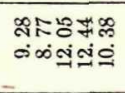 & 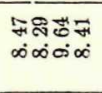 & 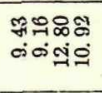 \\
\hline 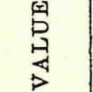 & & 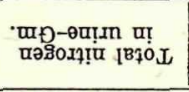 & 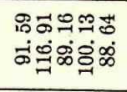 & 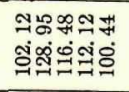 & 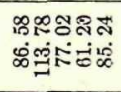 & 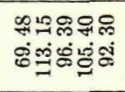 & 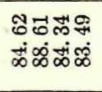 & 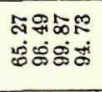 \\
\hline 번 & & 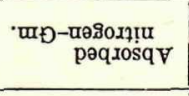 & 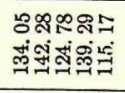 & 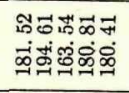 & 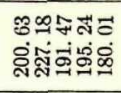 & 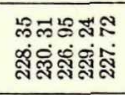 & 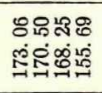 & 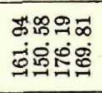 \\
\hline 음 & & 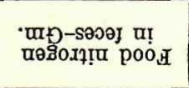 & 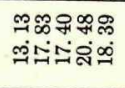 & 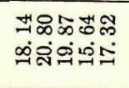 & 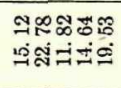 & 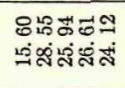 & 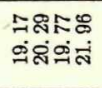 & 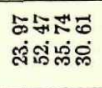 \\
\hline $\begin{array}{l}y_{1} \\
0 \\
z \\
0 \\
0\end{array}$ & & 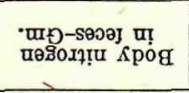 & 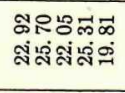 & 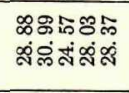 & 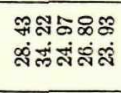 & 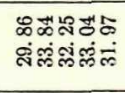 & 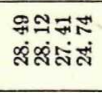 & 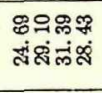 \\
\hline 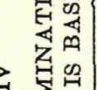 & & 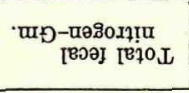 & 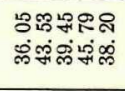 & 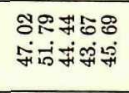 & 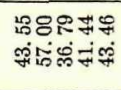 & 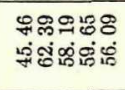 & 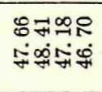 & 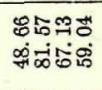 \\
\hline 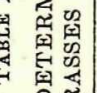 & & 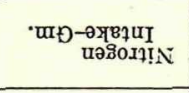 & 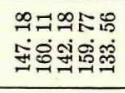 & 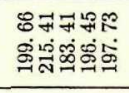 & 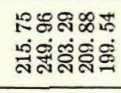 & 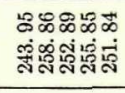 & 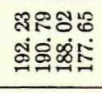 & 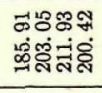 \\
\hline 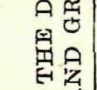 & & 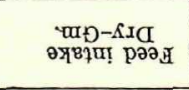 & 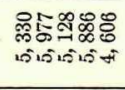 & 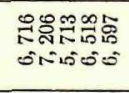 & 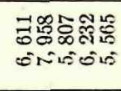 & 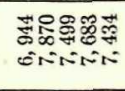 & 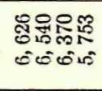 & 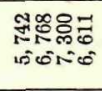 \\
\hline $\begin{array}{l}\text { 田 } \\
0 \\
\text { 苗 } \\
\end{array}$ & & 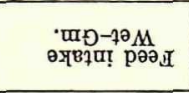 & 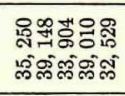 & 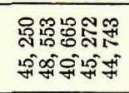 & 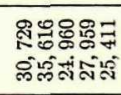 & 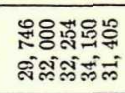 & 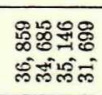 & 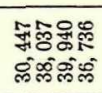 \\
\hline $\begin{array}{l}b^{2} \\
\text { z } \\
0 \\
0 \\
0\end{array}$ & 몽 & 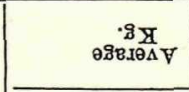 & 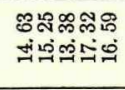 & 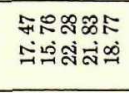 & 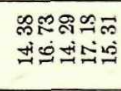 & 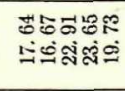 & 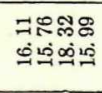 & 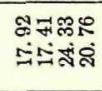 \\
\hline 点 & $\begin{array}{l}\frac{\infty}{0} \\
\overrightarrow{0} \\
0\end{array}$ & $\begin{array}{l}\text { [8प } \\
\text { [8] }\end{array}$ & 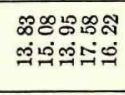 & 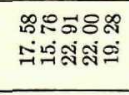 & 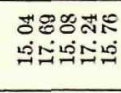 & 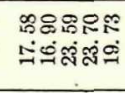 & 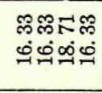 & 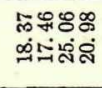 \\
\hline 蚛 & ตீ & $\begin{array}{l}\text { 98 } \\
\text { [8!7!एा }\end{array}$ & 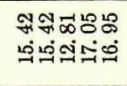 & 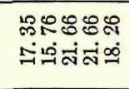 & 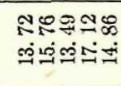 & 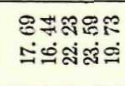 & 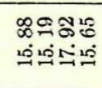 & 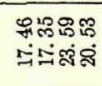 \\
\hline 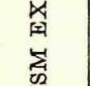 & & $\cdot \mathrm{oN}_{\mathrm{N}}$ IeuțuY & 뮤: & 쿼 ঞ夺 & ニำล & 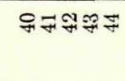 & 또조ำ & 유:웍 \\
\hline 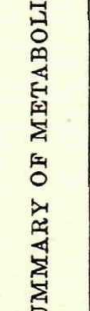 & & 莺 & 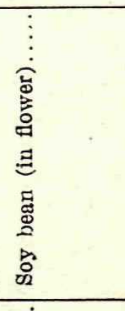 & 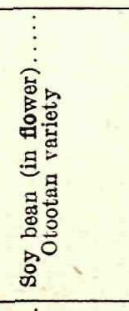 & 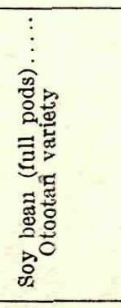 & 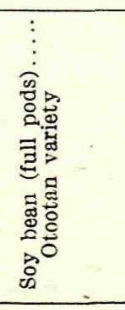 & 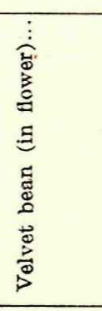 & 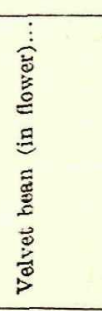 \\
\hline$p_{0}$ & & ${ }^{\circ} \mathrm{N} I$ & के & $\dot{\forall}$ & 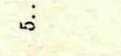 & $\dot{\infty}$ & $\therefore$ & $\dot{\infty}$ \\
\hline
\end{tabular}




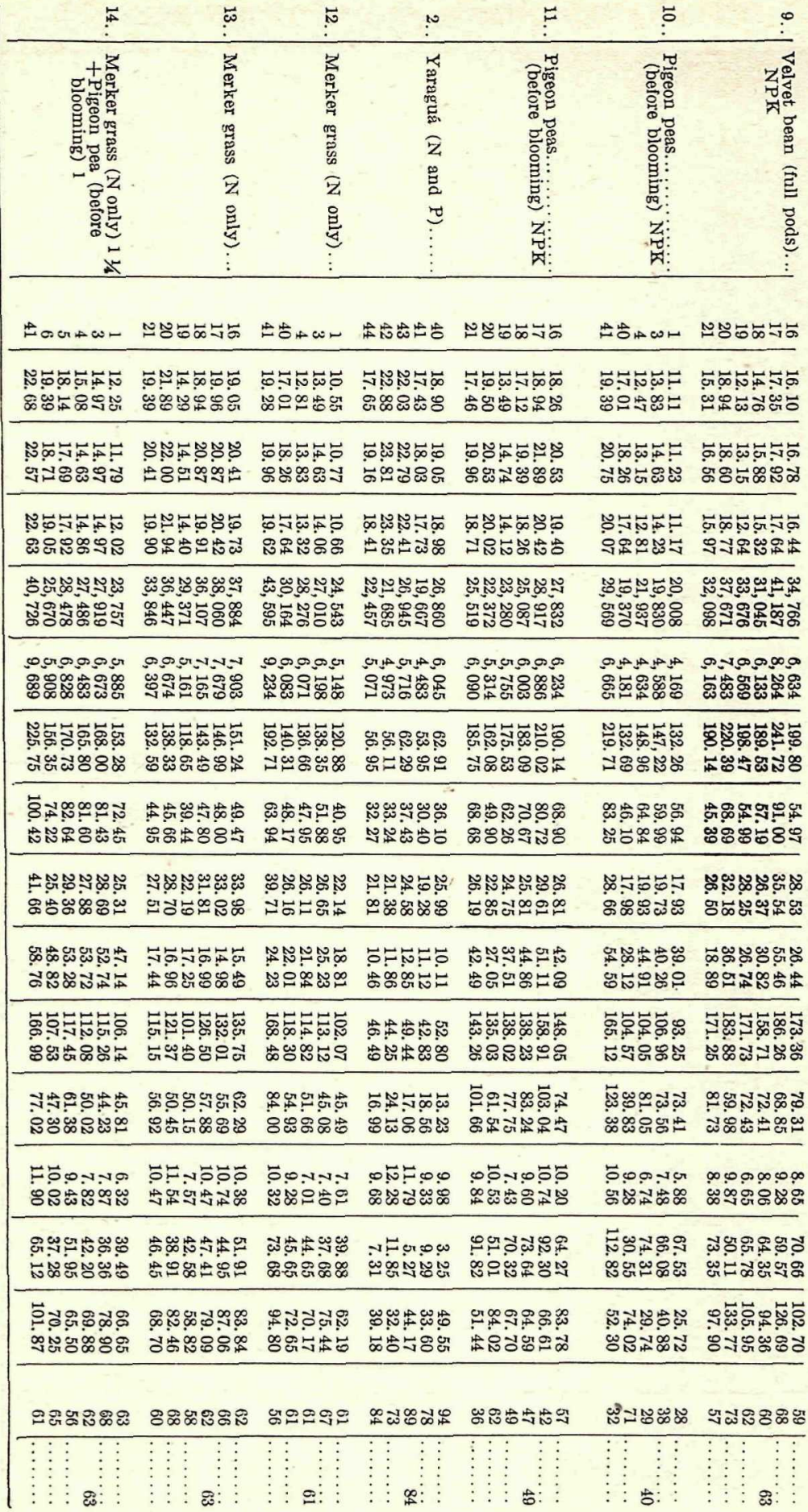


THE JOURNAL OF AGRICULTURE OF THE UNIVERSTTY OF P. $R$.

TABLE V

ANALYSES OF GRASSES AND LEGUMES

FED

WE'T BASIS

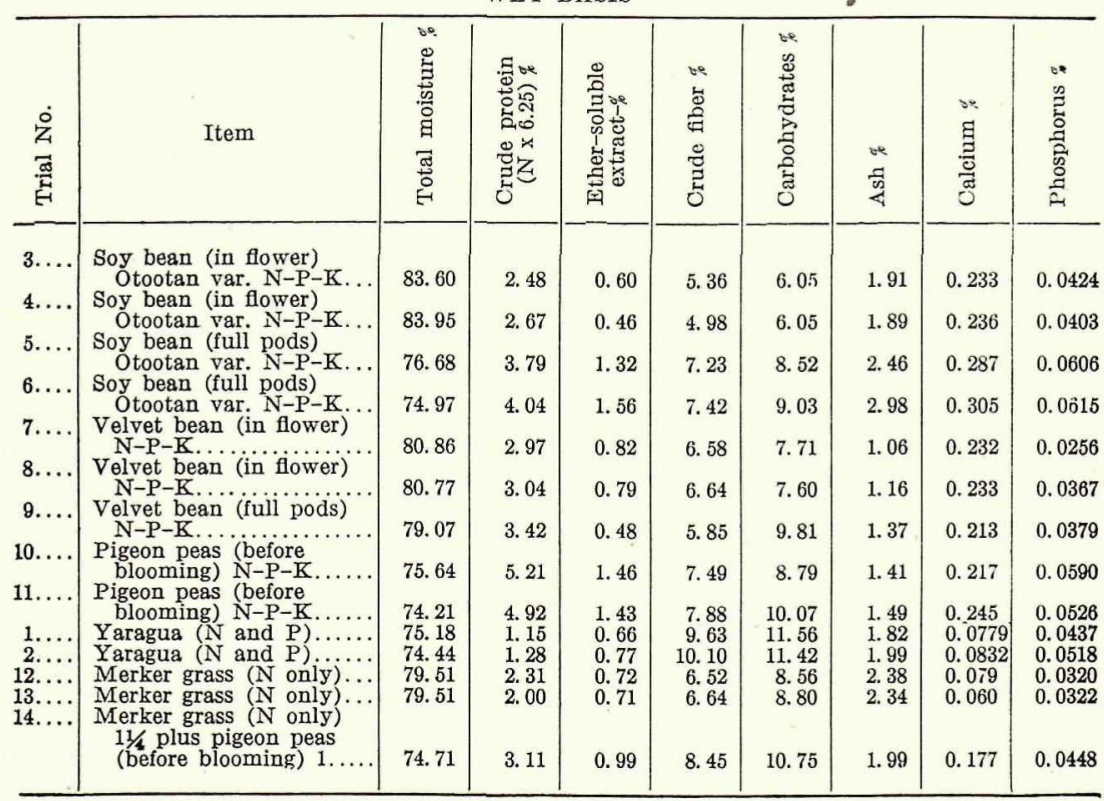


TABLE VI

ANALYSES OF GRASSES AND LEGUMES

FED

DRY BASIS

\begin{tabular}{|c|c|c|c|c|c|c|c|c|c|}
\hline 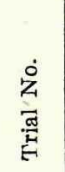 & Item & 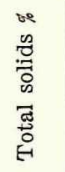 & 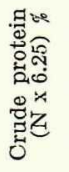 & 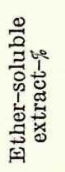 & 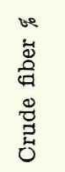 & 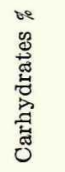 & $\begin{array}{l}0 \\
\frac{1}{4} \\
4\end{array}$ & 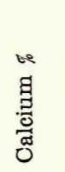 & 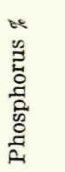 \\
\hline \multirow{17}{*}{$\begin{array}{c}3 \ldots \ldots \\
4 \ldots \\
5 \ldots \ldots \\
6 \ldots \\
7 \ldots \\
8 \ldots \\
9 \ldots \\
10 \ldots \\
11 \ldots \\
1 \ldots \\
2 \ldots \\
12 \ldots \\
13 \ldots \\
14 \ldots\end{array}$} & \multirow{17}{*}{ 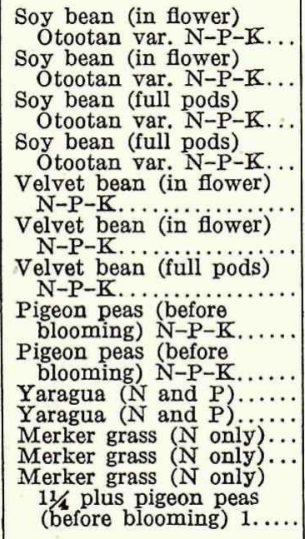 } & & & & & & & & \\
\hline & & 16. 40 & 15.13 & 3. 63 & 32.70 & 36.88 & 11. 66 & 1.42 & 0.259 \\
\hline & & 16. 05 & 16.63 & 2. 84 & 31.05 & 37.70 & 11.78 & 1.47 & 0.251 \\
\hline & & 23. 32 & 16.25 & 5.65 & 31.00 & 36.57 & 10.53 & 1.23 & 0.260 \\
\hline & & 25. 03 & 16. 12 & 6. 22 & 29.65 & 36.11 & 11.90 & 1.22 & 0.245 \\
\hline & & & & & & & & & \\
\hline & & 19. 14 & 15.51 & 4.27 & 34.38 & 40.29 & 5.55 & 1.21 & 0.134 \\
\hline & & 19. 23 & 15.80 & 4. 10 & 34.56 & 39.53 & 6.01 & 1.21 & 0.191 \\
\hline & & 20.93 & 16.36 & 2. 31 & 27. 95 & 46.84 & 6.54 & 1.02 & 0.181 \\
\hline & & 24.36 & 21.38 & 6.00 & 30.75 & 36.11 & 5. 76 & 0.89 & 0.242 \\
\hline & & & & & & & & & \\
\hline & & 24.82 & 4. 63 & 2.64 & 38.80 & 46.61 & $\begin{array}{l}7.32 \\
\end{array}$ & 0.314 & 0.176 \\
\hline & & 25.56 & 5. 00 & 3. & 39. & 44.74 & $\begin{array}{r}7.77 \\
\end{array}$ & $\begin{array}{l}0.326 \\
\end{array}$ & 0.203 \\
\hline & & 20.49 & 11.25 & 3. & 31. & 41. & 11. & 0.378 & 0.156 \\
\hline & & 20.49 & 9.75 & 3.47 & 32.40 & 42.98 & 11.43 & 0.294 & 0.157 \\
\hline & & & & & & & & & \\
\hline & & 25. 29 & 12.30 & 3. 91 & 33.45 & 42.49 & 7.85 & 0.70 & 0.177 \\
\hline
\end{tabular}


472 THE JOURNAL OF AGRICULTURE OF THE UNIVERSITY OF P. R.

\begin{tabular}{|c|c|c|c|c|c|c|c|c|c|c|c|c|c|c|c|}
\hline \multirow{4}{*}{\multicolumn{2}{|c|}{ 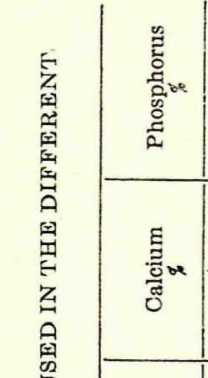 }} & 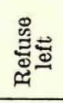 & 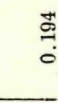 & $\stackrel{\mathscr{\Xi}}{\circ}$ & 尔 & 胥 & $\stackrel{5}{3}$ & 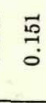 & $\stackrel{\substack{\infty \\
\hdashline \\
0}}{0}$ & 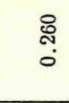 & 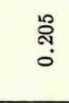 & 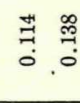 & 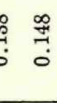 & $\begin{array}{l}\stackrel{10}{0} \\
0 \\
0\end{array}$ & $\stackrel{1}{1}$ \\
\hline & & 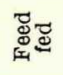 & 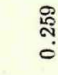 & 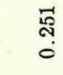 & 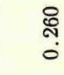 & 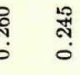 & 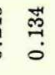 & వే & $\begin{array}{l}\vec{\infty} \\
\stackrel{0}{0}\end{array}$ & స્ّ & 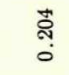 & 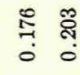 & 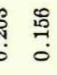 & 总 & : \\
\hline & & 总 & & 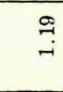 & $\stackrel{\mathbb{J}}{-}$ & సี & 菅 & $\stackrel{8}{-}$ & $\stackrel{\mathscr{\leftrightarrow}}{\longrightarrow}$ & $\begin{array}{l}\overrightarrow{5} \\
\dot{0}\end{array}$ & $\begin{array}{l}\infty \\
\infty \\
0 \\
0\end{array}$ & 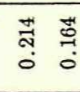 & 䓵 & 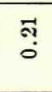 & I. \\
\hline & & 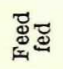 & F & $\stackrel{5}{\mathrm{~F}}$ & $\stackrel{\mathscr{q}}{-}$ & สุ & $\stackrel{\text { ஸุ }}{\text { - }}$ & 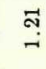 & 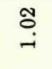 & $\begin{array}{l}\stackrel{8}{\circ} \\
0 \\
0\end{array}$ & $\begin{array}{l}\stackrel{8}{\circ} \\
0\end{array}$ & 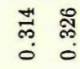 & 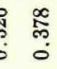 & $\begin{array}{l}\text { 芯 } \\
\stackrel{0}{0}\end{array}$ & ? \\
\hline $\begin{array}{l}0 \\
\text { n } \\
Z \\
0\end{array}$ & \multirow{2}{*}{ पू్ } & 总芯 & $\begin{array}{l}\text { \&̊ } \\
\text { తి }\end{array}$ & $\begin{array}{l}\stackrel{0}{0} \\
\text { త్ }\end{array}$ & 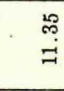 & $\begin{array}{l}\overrightarrow{0} \\
\text { త్ }\end{array}$ & สุ & ثี & స్ & :্ & $\begin{array}{l}\infty \\
\infty \\
\vdots\end{array}$ & 유 윰 & $\begin{array}{ll}0 \\
0\end{array}$ & $\begin{array}{l}\stackrel{\varnothing}{0} \\
\stackrel{\infty}{\circ}\end{array}$ & $\begin{array}{l}\text { 我 } \\
\text { is }\end{array}$ \\
\hline 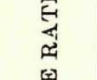 & & 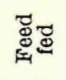 & $\stackrel{8}{=}$ & $\stackrel{\infty}{\stackrel{\infty}{=}}$ & 哭 & \& & 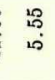 & ö & 苫 & $\stackrel{8}{?}$ & $\underset{R}{R}$ & 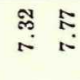 & $\stackrel{\overrightarrow{0}}{=}$ & $\stackrel{\Re}{=}$ & $\stackrel{\infty}{\infty}$ \\
\hline 肪 & \multirow{2}{*}{ 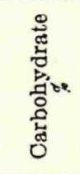 } & 总总 & कृ & 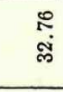 & $\begin{array}{l}\stackrel{2}{0} \\
\text { 吾 }\end{array}$ & 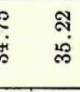 & $\begin{array}{l}\infty \\
\infty \\
\stackrel{\infty}{\infty}\end{array}$ & 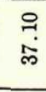 & 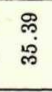 & $\begin{array}{l}\text { \& } \\
\text { జิ }\end{array}$ & $\begin{array}{l}\stackrel{2}{-1} \\
\stackrel{0}{0}\end{array}$ & 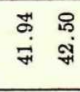 & 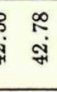 & 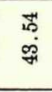 & $\begin{array}{l}\text { 足 } \\
\text { o }\end{array}$ \\
\hline 学 & & " & $\begin{array}{l}\infty \\
\infty \\
\dot{\oplus}\end{array}$ & ?̊. & 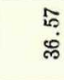 & $\begin{array}{l}\exists \\
\dot{\infty}\end{array}$ & $\begin{array}{l}\text { \& } \\
\text { †े }\end{array}$ & $\begin{array}{l}\mathscr{ஜ} \\
\text { क् }\end{array}$ & 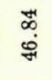 & $\begin{array}{l}\exists \\
= \\
0\end{array}$ & $\begin{array}{l}\text { के } \\
\text { के }\end{array}$ & 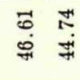 & 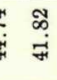 & $\begin{array}{l}\infty \\
\stackrel{\$}{*}\end{array}$ & $\begin{array}{l}\stackrel{9}{~} \\
\text { gु }\end{array}$ \\
\hline 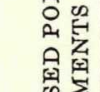 & \multirow{2}{*}{ 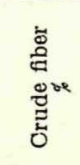 } & 荧司 & 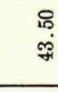 & $\begin{array}{l}\text { i. } \\
\text { 9. } \\
9\end{array}$ & $\begin{array}{l}\text { 号 } \\
\text { 겨 }\end{array}$ & $\begin{array}{l}\text { 号 } \\
\text { ㄱ. }\end{array}$ & $\begin{array}{l}\text { 유 } \\
\text { ㅁ }\end{array}$ & $\begin{array}{l}\text { 吕 } \\
\text { 方 }\end{array}$ & $\begin{array}{l}\stackrel{0}{0} \\
\stackrel{4}{*}\end{array}$ & 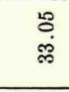 & $\begin{array}{l}\text { मे } \\
\text { में }\end{array}$ & $\begin{array}{ll}8 & 8 \\
0 & 0 \\
i & 0\end{array}$ & $\begin{array}{l}5 \\
0 \\
0\end{array}$ & $\begin{array}{l}\stackrel{\infty}{0} \\
\stackrel{\infty}{\infty}\end{array}$ & $\begin{array}{l}\mathscr{g} \\
\stackrel{9}{\$}\end{array}$ \\
\hline 电 & & 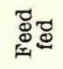 & $\begin{array}{l}\text { R } \\
\text { बूं }\end{array}$ & 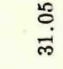 & लि & $\begin{array}{l}\text { : } \\
\text { వें }\end{array}$ & $\begin{array}{l}\infty \\
\infty \\
\text { జ }\end{array}$ & 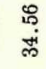 & $\begin{array}{l}\stackrel{2}{\circ} \\
\stackrel{A}{A}\end{array}$ & $\begin{array}{l}\text { ำ } \\
\text { 官 }\end{array}$ & $\begin{array}{l}\text { : } \\
\text { 官 } \\
\text { r. }\end{array}$ & $\begin{array}{ll}\infty & 0 \\
\infty & 0 \\
\infty & 0 \\
\infty & 0\end{array}$ & $\begin{array}{l}\infty \\
\dot{m} \\
\dot{m} \\
\dot{m}\end{array}$ & $\begin{array}{l}\text { 유 } \\
\text { ్ㅐ }\end{array}$ & 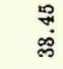 \\
\hline 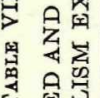 & \multirow{2}{*}{ 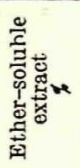 } & 总: & $\stackrel{\mathcal{Z}}{-}$ & $\overrightarrow{-}$ & $\begin{array}{l}: \\
\stackrel{8}{\circ}\end{array}$ & สุ & : & 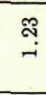 & $\underset{\sim}{\stackrel{m}{a}}$ & 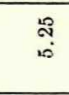 & 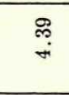 & 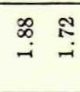 & $\begin{array}{l}\vec{m} \\
\vec{i}\end{array}$ & 品 & $\underset{\text { Ș }}{\stackrel{9}{4}}$ \\
\hline 国 & & 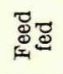 & $\begin{array}{l}\mathscr{8} \\
\stackrel{\circ}{\circ}\end{array}$ & $\begin{array}{l}\overrightarrow{0} \\
\text { ì } \\
\text { in }\end{array}$ & $\begin{array}{l}18 \\
10\end{array}$ & $\begin{array}{l}\text { స̃ } \\
\text { }\end{array}$ & 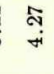 & $\stackrel{7}{7}$ & $\overrightarrow{\tilde{i}}$ & $\begin{array}{l}8 \\
0\end{array}$ & $\begin{array}{l}\text { in } \\
\text { î }\end{array}$ & $\begin{array}{ll}\overrightarrow{0} & \mathscr{0} \\
\text { o }\end{array}$ & 官 & $\underset{\infty}{\vec{n}}$ & $\vec{\sigma}$ \\
\hline 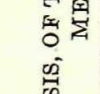 & \multirow{2}{*}{ 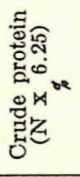 } & 总: & 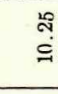 & $\begin{array}{l}\stackrel{\Re}{9} \\
\stackrel{9}{9}\end{array}$ & $\begin{array}{l}: \\
\stackrel{8}{\circ}\end{array}$ & $\begin{array}{l}\mathscr{0} \\
\dot{0}\end{array}$ & $\stackrel{\Re}{\sharp}$ & 苸 & $\begin{array}{l}\overrightarrow{0} \\
\underset{]}{J}\end{array}$ & $\begin{array}{l}\vec{m} \\
\text { สู }\end{array}$ & : & 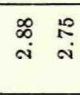 & $\underset{\infty}{\vec{p}}$ & i⿱口 & . \\
\hline$\not$ & & 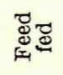 & 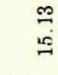 & $\begin{array}{l}\mathscr{8} \\
\stackrel{0}{\oplus}\end{array}$ & $\begin{array}{l}\stackrel{2}{0} \\
\stackrel{0}{0}\end{array}$ & $\begin{array}{l}\stackrel{2}{7} \\
\stackrel{0}{0}\end{array}$ & 占 & $\begin{array}{l}\infty \\
\dot{0} \\
\stackrel{9}{-1}\end{array}$ & $\begin{array}{l}\stackrel{\circ}{0} \\
\stackrel{\oplus}{\oplus}\end{array}$ & $\begin{array}{l}\infty \\
\stackrel{\sim}{\sim} \\
\vec{\mu}\end{array}$ & $\begin{array}{l}\stackrel{8}{0} \\
\stackrel{\square}{9}\end{array}$ & $\begin{array}{ll}\mathscr{8} & 8 \\
ن \\
+ & 0\end{array}$ & : & $\stackrel{2}{2}$ & $\begin{array}{l}\text { क् } \\
\text { 거 }\end{array}$ \\
\hline$A$ & \multirow{2}{*}{ 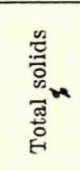 } & 递苛 & $\begin{array}{l}8 \\
\text { สं }\end{array}$ & $\begin{array}{l}\stackrel{0}{0} \\
\text { జె }\end{array}$ & : & ๙ิ & $\begin{array}{l}\overrightarrow{0} \\
\infty \\
\stackrel{0}{0}\end{array}$ & 栾 & $\underset{\mathscr{\sim}}{\tilde{\oplus}}$ & 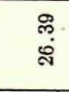 & $\begin{array}{l}\mathscr{8} \\
\text { À }\end{array}$ & \begin{tabular}{ll}
$\exists$ & \multicolumn{1}{l}{} \\
$\infty$ & 0 \\
$\infty$ & 0
\end{tabular} & 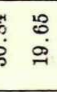 & 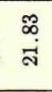 & $\begin{array}{l}\stackrel{0}{\infty} \\
\dot{\mathbf{s}}\end{array}$ \\
\hline 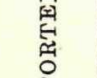 & & 焉 & ?. & $\stackrel{8}{\stackrel{8}{0}}$ & $\begin{array}{l}\text { वै: } \\
\text { वें }\end{array}$ & $\begin{array}{l}\text { \% } \\
\text { வें }\end{array}$ & 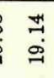 & 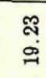 & 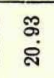 & 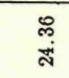 & 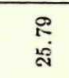 & 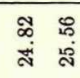 & $\begin{array}{l}9 \\
0 \\
5 \\
5 \\
5\end{array}$ & 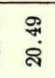 & $\begin{array}{l}\text { कి } \\
\text { జై }\end{array}$ \\
\hline 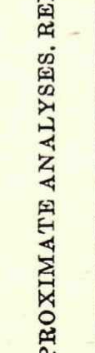 & \multicolumn{2}{|c|}{ 离 } & 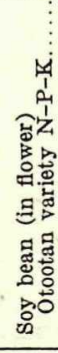 & 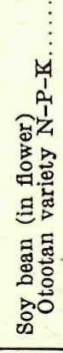 & 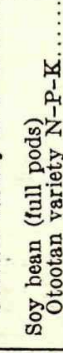 & 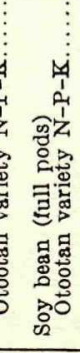 & 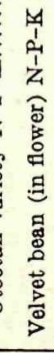 & 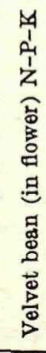 & 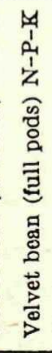 & 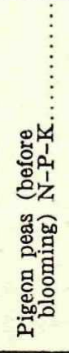 & 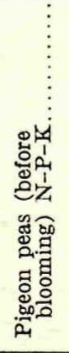 & 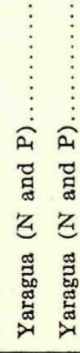 & 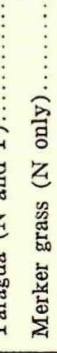 & 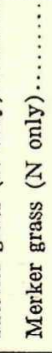 & 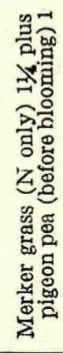 \\
\hline & & & & & & & $\therefore$ & & & & $\begin{array}{l}\vdots \\
\vdots \\
-1\end{array}$ & $\begin{array}{r}\vdots \\
\vdots \\
\vdots\end{array}$ & $\therefore$ & $\stackrel{m}{-1}$ & -7 \\
\hline
\end{tabular}




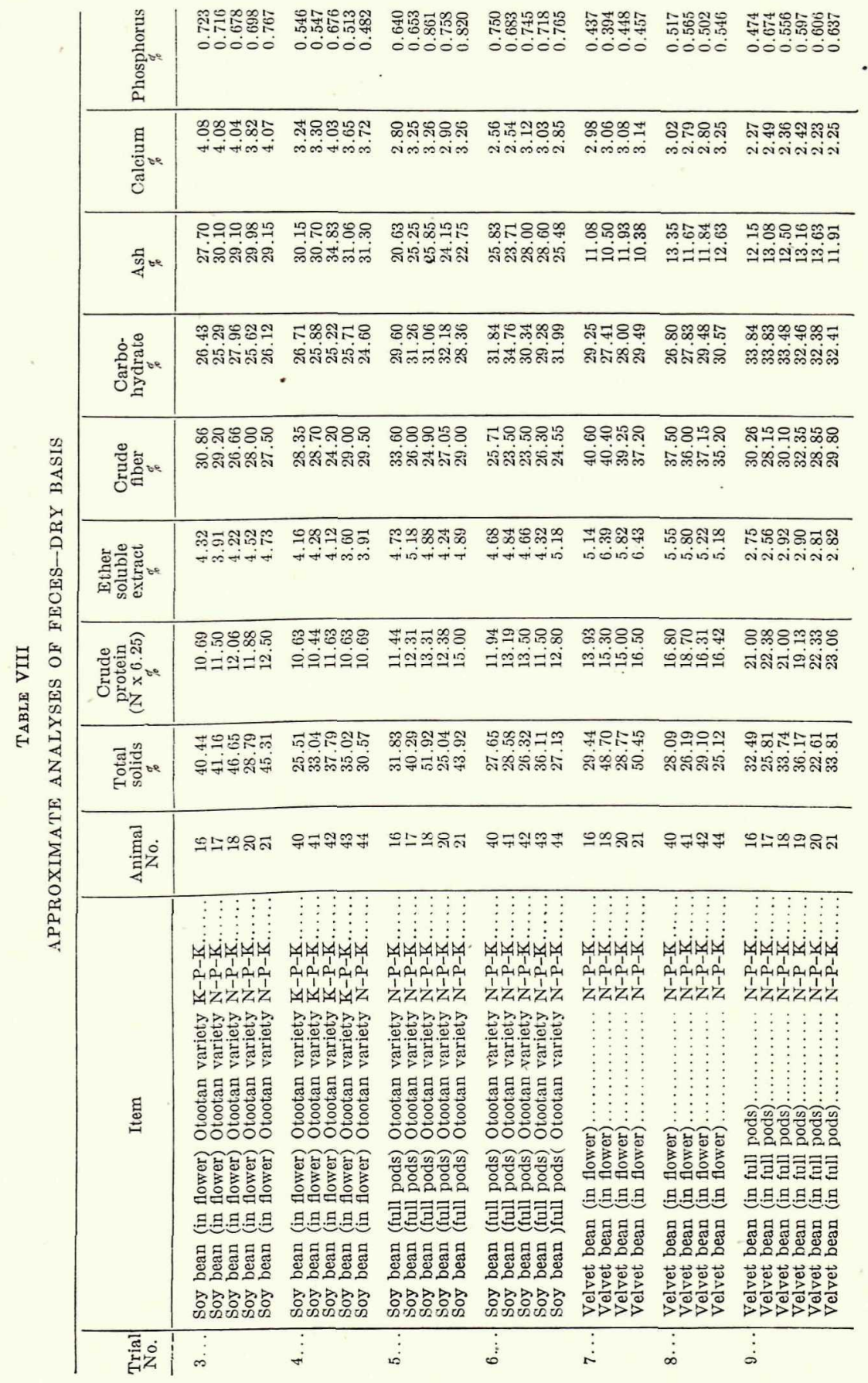




\begin{tabular}{|c|c|c|c|c|c|}
\hline 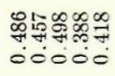 & 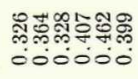 & 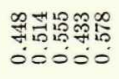 & 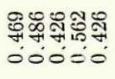 & 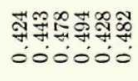 & 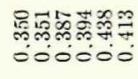 \\
\hline & & & & & \\
\hline
\end{tabular}

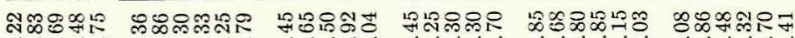

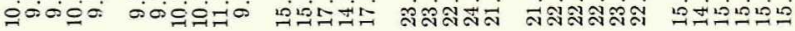

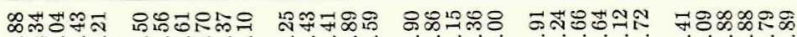

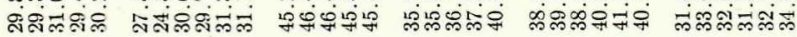

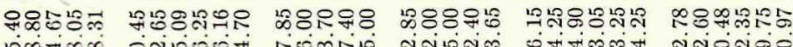
๓๓

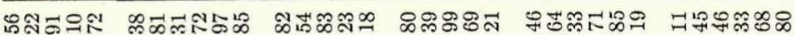

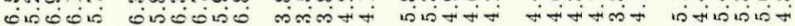

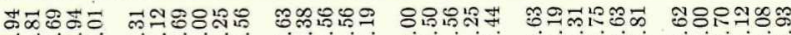
ப⿻上丨

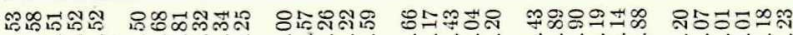

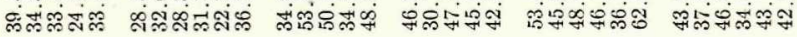

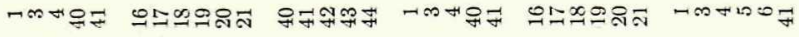

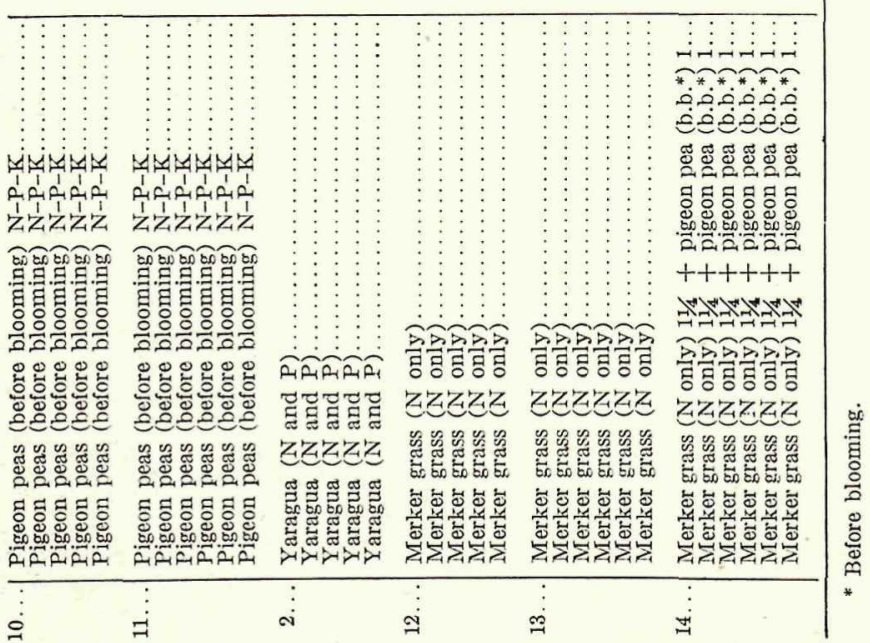


THE .JOURNAL OF AGRICULTUKE OF THE UNIVERSITY OF $ঙ$. $R$.

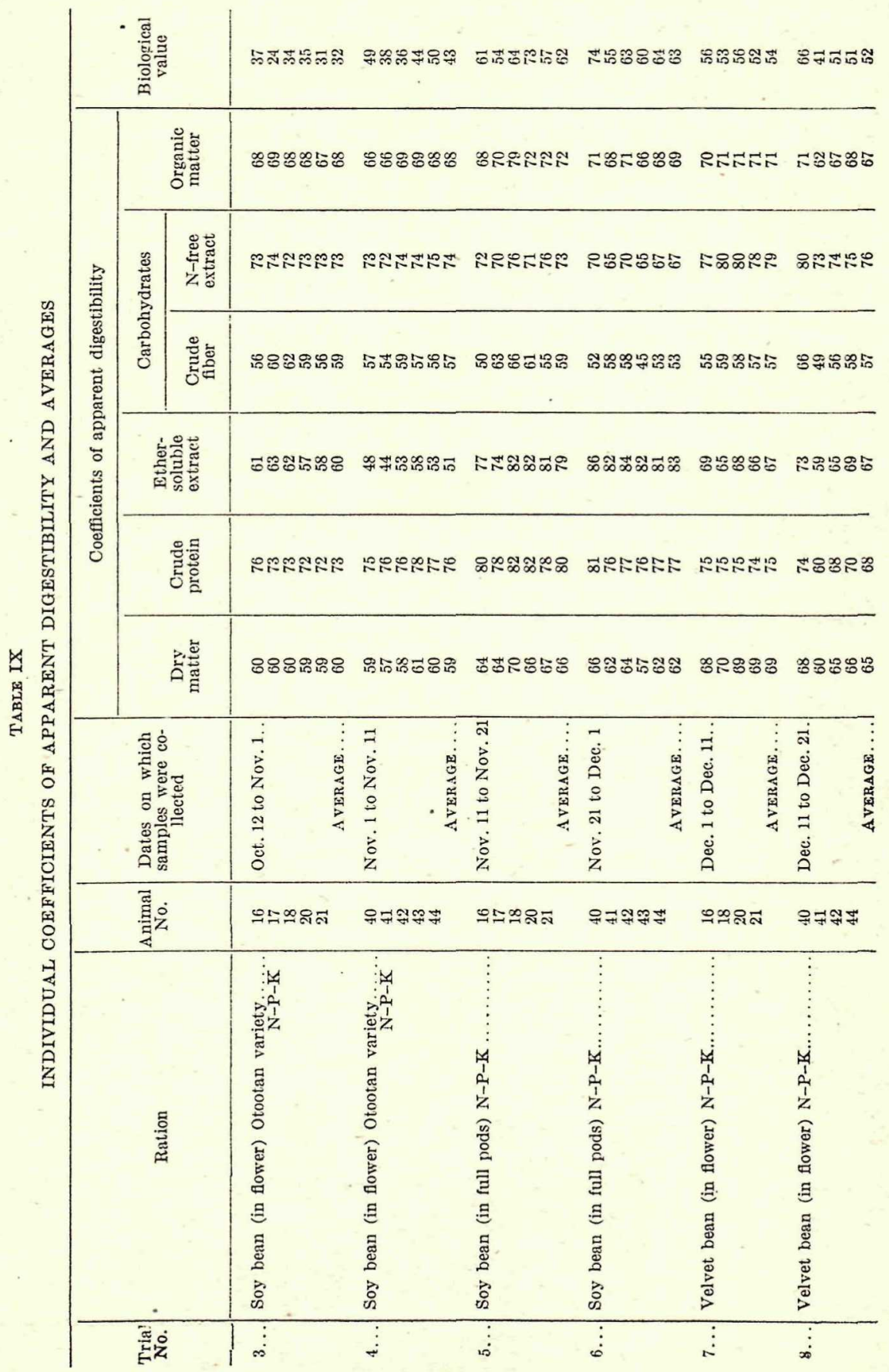




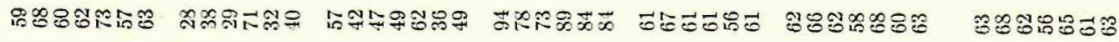

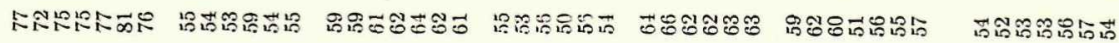

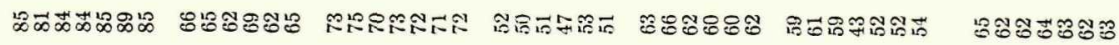

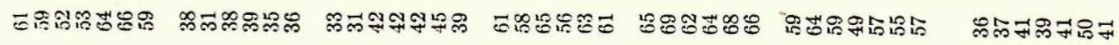

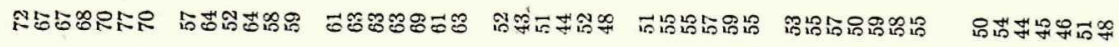

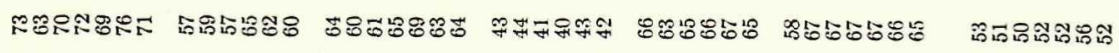

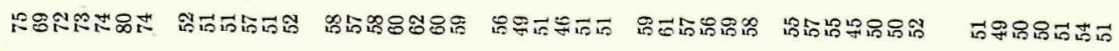

\begin{tabular}{|c|c|c|c|c|c|c|c|c|}
\hline 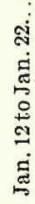 & 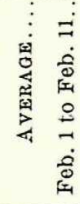 & 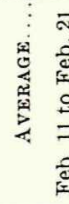 & 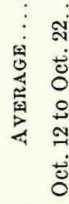 & 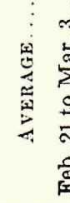 & 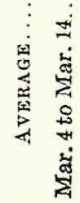 & 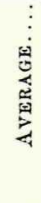 & 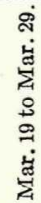 & 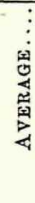 \\
\hline
\end{tabular}

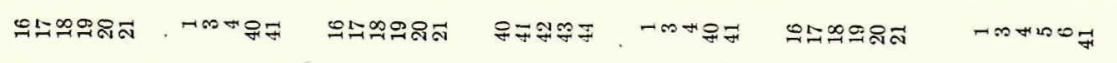

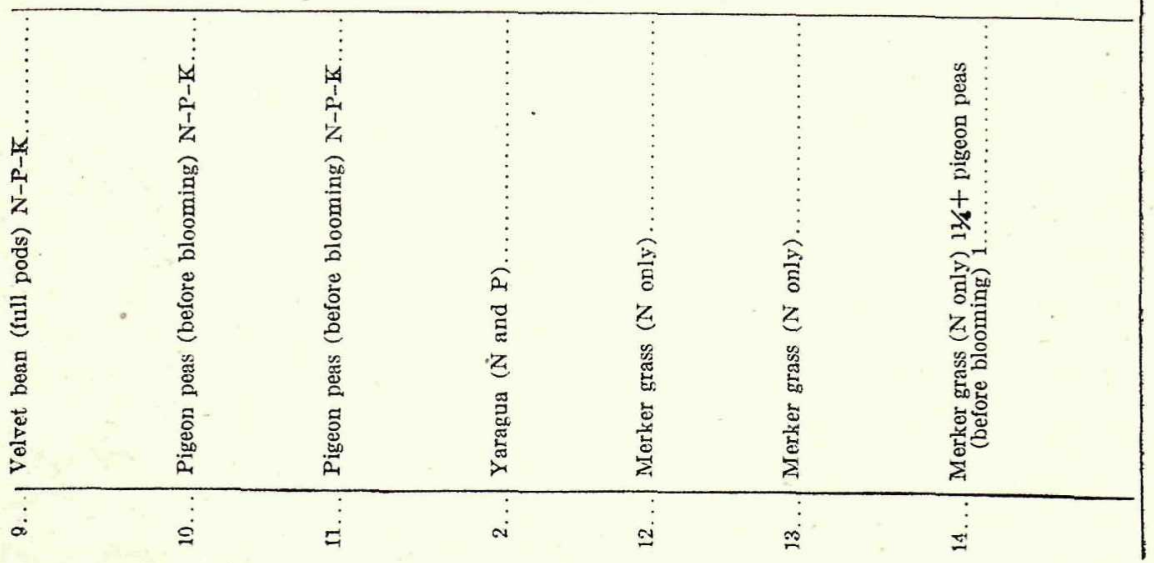




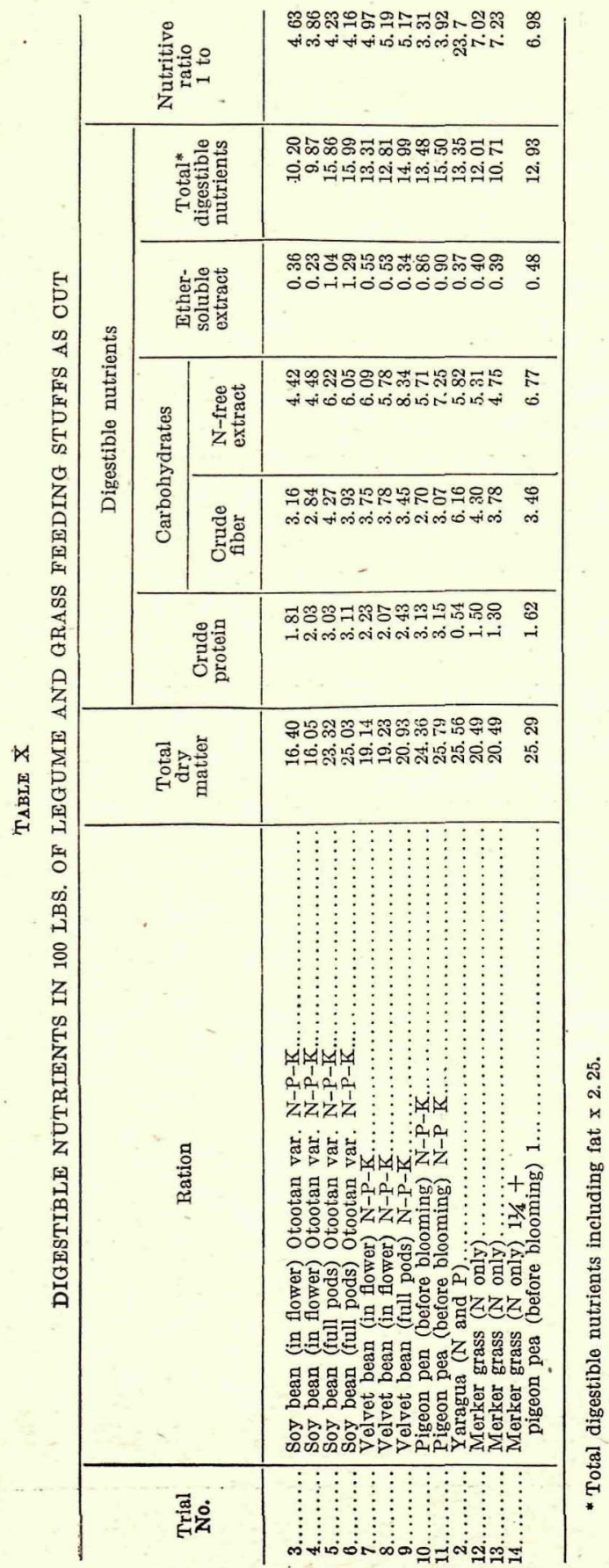


TABLE 文I

DIGESTIBLE NUTRIENTS PRODUCED PER CUERDA* OF GREEN LEGUMES**

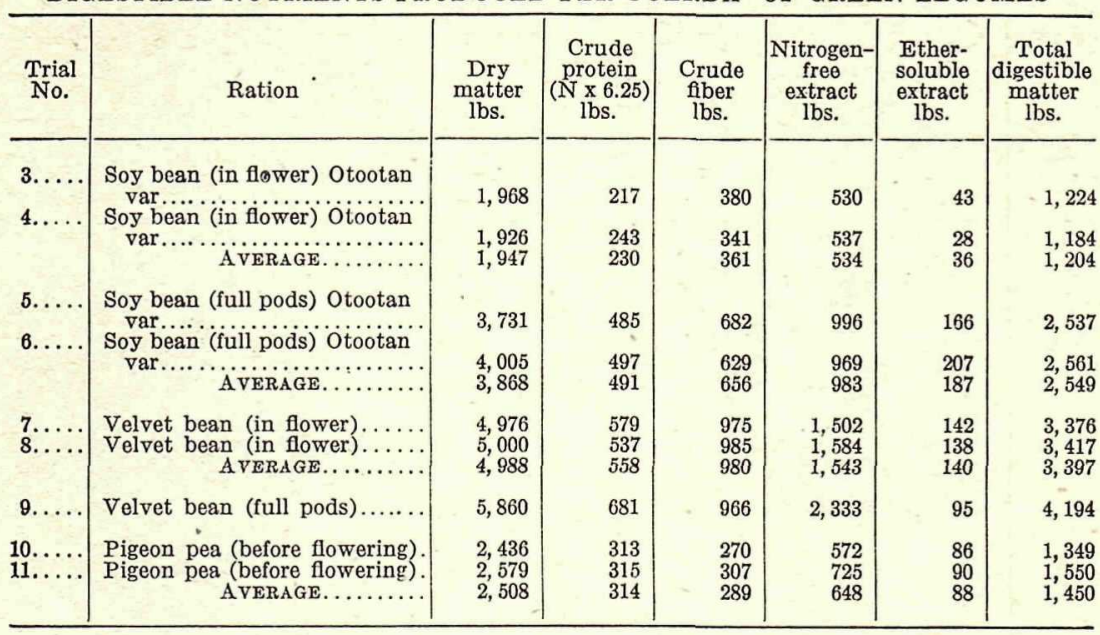

* One cuerda equals 0.954 acre.

**All fertilized with $\mathrm{N}-\mathrm{P}-\mathrm{K}$.

TABLE XII

NET PROTEIN VALUES OF COMMON LEGUMES FED SINGLY AND IN COMBINATION TO LAMBS WET BASIS

\begin{tabular}{|c|c|c|c|c|c|c|}
\hline $\begin{array}{l}\text { Trial } \\
\text { No. }\end{array}$ & Ration & 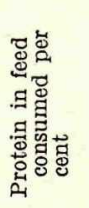 & 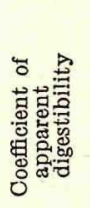 & 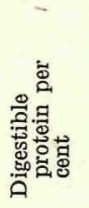 & 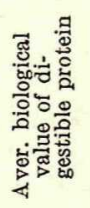 & 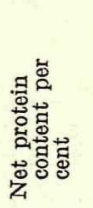 \\
\hline $\begin{array}{l}3 \ldots \ldots \\
4 \ldots \ldots\end{array}$ & 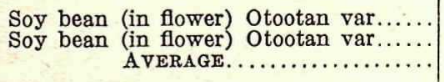 & $\begin{array}{l}2.48 \\
2.67 \\
2.58\end{array}$ & $\begin{array}{l}73 \\
76 \\
75\end{array}$ & $\begin{array}{l}1.81 \\
2.03 \\
1.92\end{array}$ & $\begin{array}{l}32 \\
43 \\
38\end{array}$ & $\begin{array}{l}0.579 \\
0.873 \\
0.726\end{array}$ \\
\hline $6 \ldots \ldots$ & 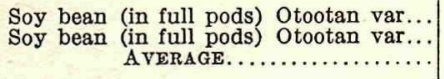 & $\begin{array}{l}\text { 3. } 79 \\
\text { 4. } 04 \\
\text { 3. } 92\end{array}$ & $\begin{array}{l}80 \\
77 \\
79\end{array}$ & $\begin{array}{l}3.03 \\
3.11 \\
3.07\end{array}$ & $\begin{array}{l}62 \\
63 \\
63\end{array}$ & $\begin{array}{l}1.88 \\
1.96 \\
1.92\end{array}$ \\
\hline $8 \ldots \ldots$ & 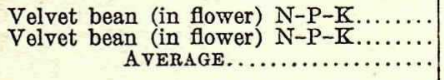 & $\begin{array}{l}2.97 \\
3.04 \\
3.01\end{array}$ & $\begin{array}{l}75 \\
68 \\
72\end{array}$ & $\begin{array}{l}2.23 \\
2.07 \\
2.15\end{array}$ & $\begin{array}{l}54 \\
52 \\
53\end{array}$ & $\begin{array}{l}1.20 \\
1.08 \\
1.14\end{array}$ \\
\hline $9 . \ldots .$. & Velvet bean (in full pods) $N-P-K . .$. & 3.42 & 71 & 2.43 & 63 & 1.53 \\
\hline $\begin{array}{l}10 . \ldots \ldots \\
11 . \ldots \ldots\end{array}$ & $\begin{array}{r}\text { Pigeon pea (before blooming) } \mathrm{N}-\mathrm{P}-\mathrm{K} . . \\
\text { Pigeon pea (before blooming) } \mathrm{N}-\mathrm{P}-\mathrm{K} . \\
\text { AvERAGE. . }\end{array}$ & $\begin{array}{l}5.21 \\
4.92 \\
5.07\end{array}$ & $\begin{array}{l}60 \\
64 \\
62\end{array}$ & $\begin{array}{l}3.12 \\
\text { 3. } 15 \\
\text { 3. } 14\end{array}$ & $\begin{array}{l}40 \\
49 \\
45\end{array}$ & $\begin{array}{l}1.25 \\
1.54 \\
1.40\end{array}$ \\
\hline $14 . \ldots$. & $\begin{array}{l}\text { Merker grass ( } N \text { only } 1=\text { plus } \\
\text { pigeon pea (before blooming) } 1 \ldots \ldots .\end{array}$ & 3.11 & 52 & 1. 62 & 63 & 1.02 \\
\hline
\end{tabular}


TABLE XIII

YIELD OF CALCIUM AND PHOSPHORUS PER CUERDA AND PER TON OF GREEN LEGUMES

\begin{tabular}{|c|c|c|c|c|c|c|c|}
\hline \multirow{2}{*}{$\begin{array}{c}\text { Trial } \\
\text { No. }\end{array}$} & \multirow{2}{*}{ Ration } & \multirow{2}{*}{$\begin{array}{c}\text { Green } \\
\text { forages } \\
\text { per } \\
\text { cuerda } \\
\text { tons* }\end{array}$} & \multirow{2}{*}{$\begin{array}{l}\text { Dry } \\
\text { legume } \\
\text { per } \\
\text { cuerda } \\
\text { lbs. }\end{array}$} & \multicolumn{2}{|c|}{ Yield per Cuerda } & \multicolumn{2}{|c|}{ Yield per Ton of } \\
\hline & & & & $\begin{array}{l}\text { Calcium } \\
\text { lbs. }\end{array}$ & $\begin{array}{l}\text { Phospho - } \\
\text { rus } \\
\text { lbs. }\end{array}$ & $\begin{array}{l}\text { Calcium } \\
\text { lbs. }\end{array}$ & $\begin{array}{l}\text { Phospho- } \\
\text { rus } \\
\text { lbs. }\end{array}$ \\
\hline $\begin{array}{l}3 \ldots \\
4 \ldots\end{array}$ & $\begin{array}{r}\text { Soy bean (in flower) Otootan var. } \\
\text { Soy bean (in flower) Otootan var. } \\
\text { AvERAGE......... }\end{array}$ & $\begin{array}{r}6 \\
6 \\
6\end{array}$ & $\begin{array}{l}1,968 \\
1,926 \\
1,947\end{array}$ & $\begin{array}{l}27.95 \\
28.31 \\
28.13\end{array}$ & $\begin{array}{l}\text { 5. } 10 \\
\text { 4. } 83 \\
\text { 4. } 96\end{array}$ & $\begin{array}{l}\text { 4. } 66 \\
\text { 4. } 72 \\
\text { 4. } 69\end{array}$ & $\begin{array}{l}0.85 \\
0.81 \\
0.83\end{array}$ \\
\hline $\begin{array}{l}5 \ldots \\
6 \ldots\end{array}$ & $\begin{array}{r}\text { Soy bean (full pods) Otootan var.. } \\
\text { Soy bean (full pods) Otootan var.. } \\
\text { AvERAGE......... }\end{array}$ & $\begin{array}{l}8 \\
8\end{array}$ & $\begin{array}{l}3,731 \\
4,005 \\
3,868\end{array}$ & $\begin{array}{l}45.89 \\
48.86 \\
47.38\end{array}$ & $\begin{array}{l}9.70 \\
9.81 \\
9.75\end{array}$ & $\begin{array}{l}5.74 \\
6.11 \\
5.92\end{array}$ & $\begin{array}{l}1.21 \\
1.23 \\
1.22\end{array}$ \\
\hline $\begin{array}{l}7 \ldots \\
8 \ldots\end{array}$ & $\begin{array}{l}\text { Velvet bean (in flower) } \ldots \ldots \ldots \ldots \\
\text { Velvet bean (in flower) } \ldots \ldots \ldots \ldots \\
\text { AvERAGE........... }\end{array}$ & $\begin{array}{r}13 \\
13 \\
\ldots \ldots\end{array}$ & $\begin{array}{l}4,976 \\
5,000 \\
4,989\end{array}$ & $\begin{array}{l}60.21 \\
60.50 \\
60.36\end{array}$ & $\begin{array}{l}6.67 \\
9.55 \\
8.04\end{array}$ & $\begin{array}{l}4.63 \\
\text { 4. } 65 \\
4.64\end{array}$ & $\begin{array}{l}0.51 \\
0.73 \\
0.62\end{array}$ \\
\hline $9, \ldots$ & Velvet bean (full pods).......... & 14 & 5,860 & 59. 77 & 10.61 & 4. 27 & 0.76 \\
\hline $\begin{array}{l}10 \ldots \\
11 \ldots\end{array}$ & $\begin{array}{r}\text { Pigeon pea (before blooming).... } \\
\text { Pigeon pea (before blooming) .... } \\
\text { AvERAGE......... }\end{array}$ & $\begin{array}{r}5 \\
5 \\
\ldots \ldots\end{array}$ & $\begin{array}{l}2,436 \\
2,579 \\
2,508\end{array}$ & $\begin{array}{l}21.68 \\
24.50 \\
23.09\end{array}$ & $\begin{array}{l}5.90 \\
5.26 \\
5.58\end{array}$ & $\begin{array}{l}\text { 4. } 34 \\
\text { 4. } 80 \\
\text { 4. } 62\end{array}$ & $\begin{array}{l}1.18 \\
1.05 \\
1.11\end{array}$ \\
\hline
\end{tabular}

*Yields obtained at the Experiment Station.

TABLE XIV

VALUE OF THE LEGUMES IN FATTENING

\begin{tabular}{|c|c|c|c|c|c|c|}
\hline Ration & $\begin{array}{c}\text { A verage } \\
\text { dry } \\
\text { matter } \\
\text { ingested } \\
\text { per head } \\
\text { Gms. }\end{array}$ & $\begin{array}{l}\text { Number } \\
\text { of } \\
\text { animals }\end{array}$ & $\begin{array}{l}\text { Number } \\
\text { of days } \\
\text { in ex- } \\
\text { periment }\end{array}$ & $\begin{array}{c}\text { Average } \\
\text { weight } \\
\text { of lamb } \\
\text { Kgms. }\end{array}$ & $\begin{array}{c}\text { Increase } \\
\text { in } \\
\text { weight } \\
\text { Kgms. }\end{array}$ & $\begin{array}{c}\text { Increase } \\
\text { in } \\
\text { weight } \\
\text { Per cent }\end{array}$ \\
\hline $\begin{array}{l}\text { Soy bean (in flower) Otootan var..... } \\
\text { Soy bean (full pods) Otootan var..... } \\
\text { Velvet bean (in flower) } \ldots \ldots \ldots \ldots \ldots \ldots \ldots \\
\text { Velvet bean (full pods) } \ldots \ldots \ldots \ldots \ldots \ldots \\
\text { Pigeon pea (before blooming) } \ldots \ldots \ldots \ldots \\
\text { Merker grass ( } N \text { only) } \ldots \ldots \ldots \ldots \ldots \\
\text { Merker grass ( } N \text { only) } 11 / 4 \text { plus } \\
\text { pigeon pea (hefore blooming) } 1 \ldots \ldots\end{array}$ & $\begin{array}{l}5,968 \\
6,960 \\
6,464 \\
6,874 \\
5,502 \\
6,701 \\
6,911\end{array}$ & $\begin{array}{r}10 \\
10 \\
8 \\
6 \\
11 \\
11\end{array}$ & $\begin{array}{l}20 \\
20 \\
20 \\
10 \\
20 \\
20\end{array}$ & $\begin{array}{l}18.38 \\
17.44 \\
17.74 \\
15.76 \\
15.70 \\
17.62 \\
16.12\end{array}$ & 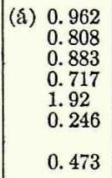 & $\begin{array}{r}5.23 \\
4.63 \\
5.02 \\
4.56 \\
12.22 \\
1.39 \\
2.94\end{array}$ \\
\hline
\end{tabular}

(a)-Average decrease in weight.

(b) - Per cent decrease in weight. 


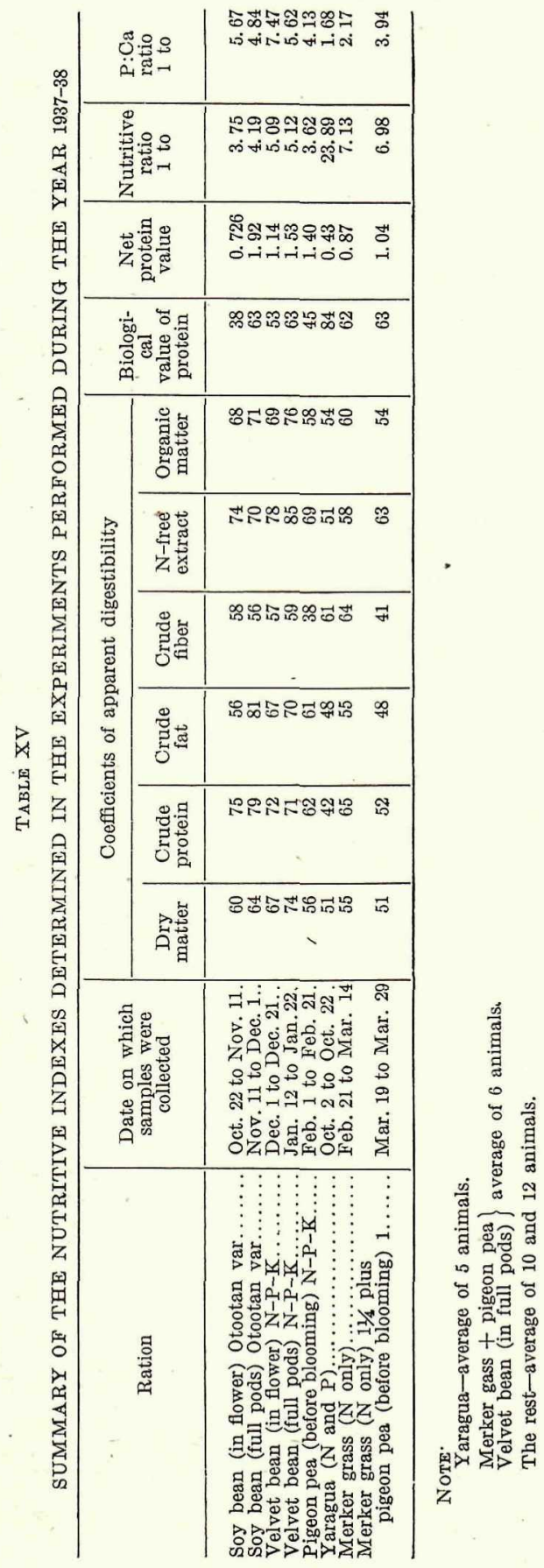

